\title{
MVAC Regimen
}

National Cancer Institute

\section{Source}

National Cancer Institute. MVAC Regimen. NCI Thesaurus. Code C63486.

A regimen consisting of methotrexate, vinblastine, doxorubicin and cisplatin used for the treatment of advanced-stage bladder and urothelial cancer. 